\title{
Characterization and Application of Poly(propylene- $b$-methyl methacrylate) as a Compatibilizer for Polypropylene/Poly(methyl methacrylate) Blend
}

\author{
Satoru Hosoda, Hayato Kinara, Keitaro Kojima, \\ Yuufu SATOH, and Yoshiharu Dor* \\ Sumitomo Chemical Co., Ltd., Chiba Research Laboratory, \\ Anesakikaigan 5-1, Ichihara-shi, Chiba 299-01, Japan \\ * Research Laboratory of Resources Utilization, Tokyo Institute of Technology, \\ Nagatsuda-cho, Midori-ku, Yokohama 227, Japan
}

(Received May 1, 1990)

\begin{abstract}
A diblock copolymer of propylene and methyl methacrylate (MMA), prepared by the living coordination polymerization of propylene followed by the subsequent polymerization of MMA, was characterized in detail, and the effect of the copolymer as a compatibilizer for the blend of isotactic polypropylene (PP) and poly(methyl methacrylate) (PMMA) was investigated. The block copolymer showed a very sharp molecular weight distribution $\left(M_{w} / M_{n}=1.1\right)$ and two glass transitions at $-3^{\circ} \mathrm{C}$ and $105^{\circ} \mathrm{C}$, which might be due to $T_{\mathrm{g}}$ of polypropylene block sequence and PMMA one, respectively. Syndiotactic sequence was found to be dominant in each block sequence. Addition of small amount of the block copolymer into PP/PMMA blends reduced both PMMA particle size and PP spherulite size. Good adhesion of the interface between PP and PMMA was achieved by the intervention of the block copolymer, resulting in a great improvement of mechanical properties of the blend.

KEY WORDS Diblock Copolymer / Micro-Phase-Separation / Polypropylene / Poly(methyl methacrylate) / Blend / Compatibilizer / Mechanical Properties /
\end{abstract}

A large number of commercial "polymer alloy" products have been continuing to make inroads into various industrial materials. Most of them are composed of immiscible polymer systems, and then, multiple phase separation is an essential feature. Among the necessary features of these immiscible polymer alloys, it is well known that good adhesion between components and adequate cross-linking are the most significant to achieve high strength. ${ }^{1,2}$ From this point of view, in commercial polymer alloy manufacturing, stabilization of interface is attempted by adding interfacially active block or graft copolymer compatibilizers, ${ }^{3-6}$ or by forming the copolymers during reactive processing in which polymers having a reactive function group are used as a compatibilizer. ${ }^{7-11}$ In the former case, the copolymer is designed so that each block or graft is miscible or partially miscible in one of the major components and immiscible in the other. Thus, the blend systems in which block copolymer is used as a compatibilizer are classified into three categories; I) major component polymers are the same as each block, II) one of the major components is the same as either block, and other component is miscible in another block, and III) both components are different from each block but miscible. Table I shows the examples of the blend systems in which A-B type diblock copolymer is used as a compatibilizer. In almost all blends shown here, the compatibilizing effect was confirmed in dispersed domain size and in some of the mechanical properties.

As shown in Table I, the styrenic copolymers 
Table I. Examples of A-B type diblock copolymer as a compatibilizer of polymer blends

\begin{tabular}{cllll}
\hline Type & Blend component & Block copolymer & Blend system & References \\
\hline & & PS-PHBd, PS-PHI & PS/LDPE & 12 \\
I & A/B & PS-PP & PS/PP & 13 \\
& & PS-PBT & PS-PBT & 15 \\
& PS-Ny6 & PS/Ny6 & 16 \\
& & PS-PMMA, PBd-PMMA & SBR/SAN & 18 \\
II & A/C & PS-PCL & PMS/PVF 2 & 19,20 \\
& & PS-PMMA & PS/PVC & 21 \\
& & PHBd-PMMA & LDPE/PVF & 22 \\
& & PS-PMMA & PPO/PVF & \\
\hline
\end{tabular}

PS, polystyrene; PHBd, hydrogenated polybutadiene; PHI, hydrogenated polyisoprene; LDPE, low-density polyethylene; PP, polypropylene; PBT, poly(butylene terephthalate); Ny6, nylon 6; PMMA, poly(methyl methacrylate); SBR, poly(styrene-co-butadiene); SAN, poly(styrene-co-acrylonitrile); PMS, poly( $\alpha$-methylstyrene); $\mathrm{PVF}_{2}, \mathrm{poly}$ (vinylidine fluoride); PCL, polycaprolactone; PVC, poly(vinyl chloride), PPO, poly(2,6-dimethyl-1,4-phenylene oxide).

are exclusively dominant in the reported diblock copolymers as a compatibilizer. This is probably due to the technical development of "living anionic polymerization" of styrenic monomers. While, the studies on the diblock copolymer of polyolefin and its application as a compatibilizer seem very few. Giudice $e t$ al. ${ }^{13}$ reported the synthesis of the diblock copolymer of propylene and styrene with the use of heterogeneous Ziegler-Natta catalyst, and elucidated the compatibilizing effect of the block copolymer for polypropylene(PP)/polystyrene(PS) blends. Block copolymer of propylene and 1,4-butadiene was obtained by Drzewinski et al. ${ }^{14}$ using a transformation from living anionic to Ziegler-Natta polymerization mechanisms. In both block copolymerizations above, only low efficiency of block copolymer formation $(15-20 \mathrm{wt} \%$ of total polymer) was obtained because of the production of homopolymers. Recently, Doi et $a .^{24-27}$ reported the syntheses of various block copolymers of propylene using living coordination polymerization of propylene with the soluble catalyst system which is followed by the polymerization of another monomer such as ethylene, tetrahydrofuran, or methyl methacrylate (MMA).
In this paper, the block copolymer of propylene and MMA was characterized in detail and then the compatibilizing effect of the copolymer was investigated for PP/PMMA blends.

\section{EXPERIMENTAL}

\section{Materials}

Poly(propylene- $b$-methyl methacrylate) studied here was prepared by the living coordination polymerization of propylene with the soluble catalyst system of tris(2-methyl-1,3butanedionate) vanadium $/ \mathrm{Al}\left(\mathrm{C}_{2} \mathrm{H}_{5}\right)_{2} \mathrm{Cl}$ at $-60^{\circ} \mathrm{C}$ in $n$-heptane and subsequently by raising temperature up to $0^{\circ} \mathrm{C}$ to polymerize MMA. Details were described in a previous paper. ${ }^{26}$ The copolymer obtained was heated in acetone at $55^{\circ} \mathrm{C}$ for 8 hours, but nothing was extracted, suggesting that homopolymer of methyl methacrylate was not produced.

\section{Polymer Blends}

Isotactic polypropylene, PP, (Sumitomo Noblen, MW $=20 \times 10^{4}$ ) and PMMA (Sumipex, $\mathrm{MW}=11 \times 10^{4}$ ) were used as components of polymer blends. Binary blends of PP and PMMA, added with the block copolymer was 
prepared by mixing each polymer in xylene solution at $120^{\circ} \mathrm{C}$, casted from the solution at $100^{\circ} \mathrm{C}$, and dried at $50^{\circ} \mathrm{C}$ for. 48 hours.

Mechanical properties; Samples were hotpress molded at $200^{\circ} \mathrm{C}$ into a sheet of $1 \mathrm{~mm}$ thickness. Tensile test was carried out at room temperature at drawing rate of $30 \mathrm{~mm} \mathrm{~min}^{-1}$. Tensile impact strength was measured following ASTM D1822 at room temperature.

\section{Characterization of Samples}

Moleculear weight and molecular weight distribution were measured with GPC (Toso HLC 811) equipped with low-angle laser light scattering photometer (Chromatix, KMX-6) at $140^{\circ} \mathrm{C}$ using trichlorobenzene as a solvent. Thermodiagram of polymers were recorded with Seiko DSC 200 system. A DSC sample of about $5 \mathrm{mg}$ was heated at $20^{\circ} \mathrm{C} \mathrm{min}^{-1}$ from -80 to $180^{\circ} \mathrm{C}$. Microtacticity and chemical composition were measured with a Bruker AM400 NMR spectrometer. Conditions for measurements for ${ }^{1} \mathrm{H}$ and ${ }^{13} \mathrm{C}$ NMR spectra are as follows: ${ }^{1} \mathrm{H}$ NMR; pulse width $4.0 \mathrm{~s}$, delay $2.0 \mathrm{~s}$, acquisition time $2.0 \mathrm{~s}$, spectral width $8064 \mathrm{~Hz} .{ }^{13} \mathrm{C}$ NMR; pulse width $4.6 \mathrm{~s}$, delay $0 \mathrm{~s}$, acquisition time $0.6 \mathrm{~s}$, spectral width $29400 \mathrm{~Hz}$, data point $32 \mathrm{~K}$.

\section{Morphology Observation}

Ultramicrotomed thin section of the block copolymer was stained by ruthenium tetroxide aqueous solution (conc. $1 \%$ ) at $60^{\circ} \mathrm{C}$ for $1 \mathrm{~h}$ and observed with a Hitachi H-8000 transmission electron microscope (TEM). Mor- phologies of the blend samples were observed with an optical microscope and a Hitachi S-4000 scanning electron microscope. The microtomed surface of the blend sample was etched by $n$-heptane at $60^{\circ} \mathrm{C}$ for $5 \mathrm{~min}$. Under this condition, only the block copolymer was soluble. Neither isotactic PP nor PMMA which were used as blend components was soluble even under the more severe condition $\left(80^{\circ} \mathrm{C}\right.$, $30 \mathrm{~min})$.

\section{RESULTS AND DISCUSSION}

\section{Characterization of Poly(propylene-b-MMA)}

Some of the characterization data of samples were shown in Table II. Molecular weight distribution of the diblock copolymer and homo-polypropylene prepared by the living coordination polymerization method were very sharp $\left(M_{w} / M_{n}=1.1\right)$, indicating one of the characteristics of "living polymer." Considering the results that no extract was obtained by the extraction with acetone and the block copolymer has very sharp molecular weight distribution similarly to homopolypropylene, no chain transfer reaction of MMA might occur during the sequential polymerization. Chemical composition of the block copolymer was calculated from ${ }^{1} \mathrm{H}$ NMR spectrum and weight ratio of polypropylene-PMMA was found to be $73: 27$. Then, molecular weight of polypropylene and PMMA sequences in the block copolymer can be calculated to be 33600 and 12400 , respectively, based on the weight average molecular weight of the copolymer.

Table II. Characteristics of polypropylene and poly(propylene- $b$-MMA) via living coordination polymerization

\begin{tabular}{|c|c|c|c|c|c|c|c|c|}
\hline \multirow{2}{*}{ Sample } & \multirow{2}{*}{$M_{w} \times 10^{-4}$} & \multirow{2}{*}{$M_{n} \times 10^{-4}$} & \multirow{2}{*}{$M_{w} / M_{n}$} & \multicolumn{2}{|c|}{$T_{\mathrm{g}}$} & \multirow{2}{*}{$\begin{array}{l}\text { Composition }^{a} \\
\text { PP/PMMA }\end{array}$} & \multicolumn{2}{|c|}{ Syndio diad fraction ${ }^{b}$} \\
\hline & & & & & & & PP block & PMMA block \\
\hline Block & 4.6 & 4.3 & 1.07 & -3.1 & 104.9 & $73 / 27$ & 79.3 & 86.2 \\
\hline Homo PP & 5.5 & 5.1 & 1.08 & -3.7 & & - & 80.6 & - \\
\hline
\end{tabular}

a Weight ratio of PP/PMMA determined by ${ }^{1} \mathrm{H}$ NMR spectrum.

b Syndiotactic fraction determined by ${ }^{13} \mathrm{C}$ NMR spectrum. 
Further, the stereo-regularity of each block sequence was determined from ${ }^{13} \mathrm{C}$ NMR spectrum, in which methylene region of polypropylene sequence and carbonyl region of PMMA sequence were used for calculation. As shown in Table II, syndiotactic sequence was found to be dominant in each block sequence though the absolute values of the stereoregularity are not so high.

DSC thermogram of the copolymer (Fig-

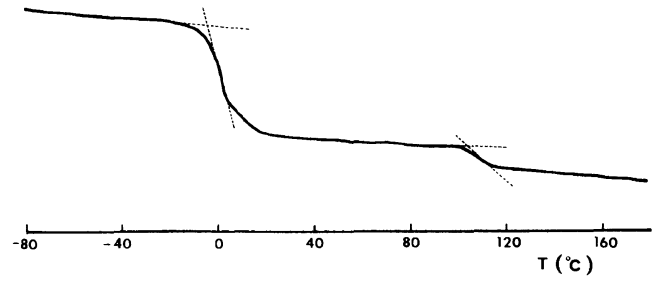

Figure 1. DSC endothermic curve of poly(propylene- $b$ MMA).

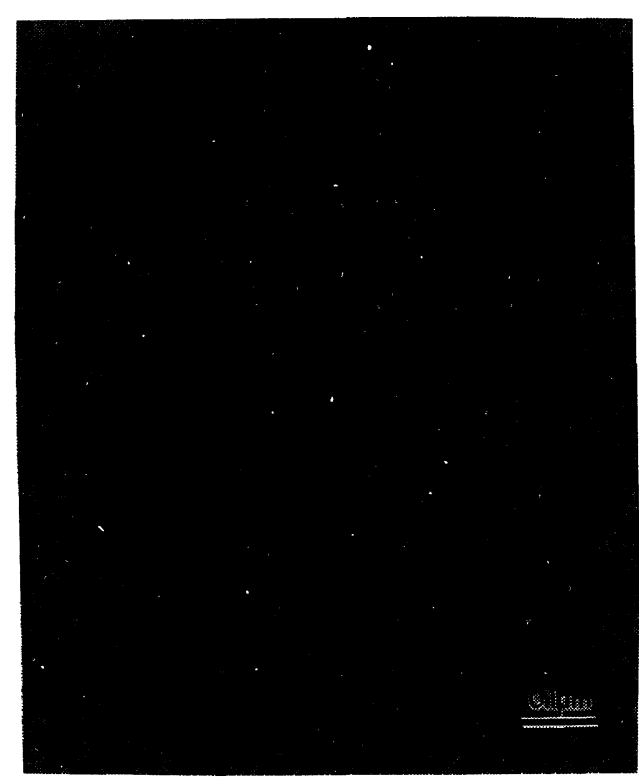

Figure 2. Transmission electron micrography of poly(propylene- $b$-MMA) stained with $\mathrm{RuO}_{4}$.

\section{A}
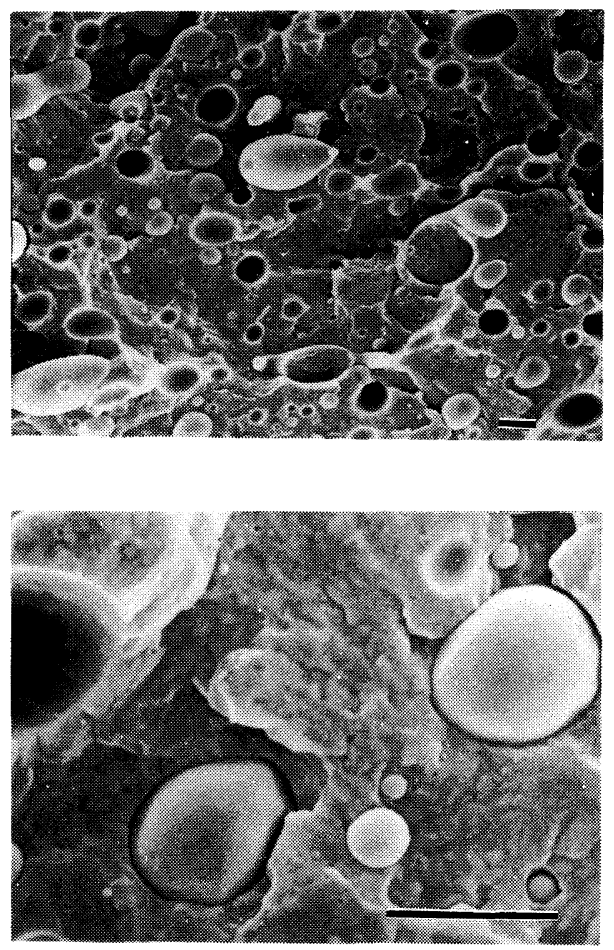

B
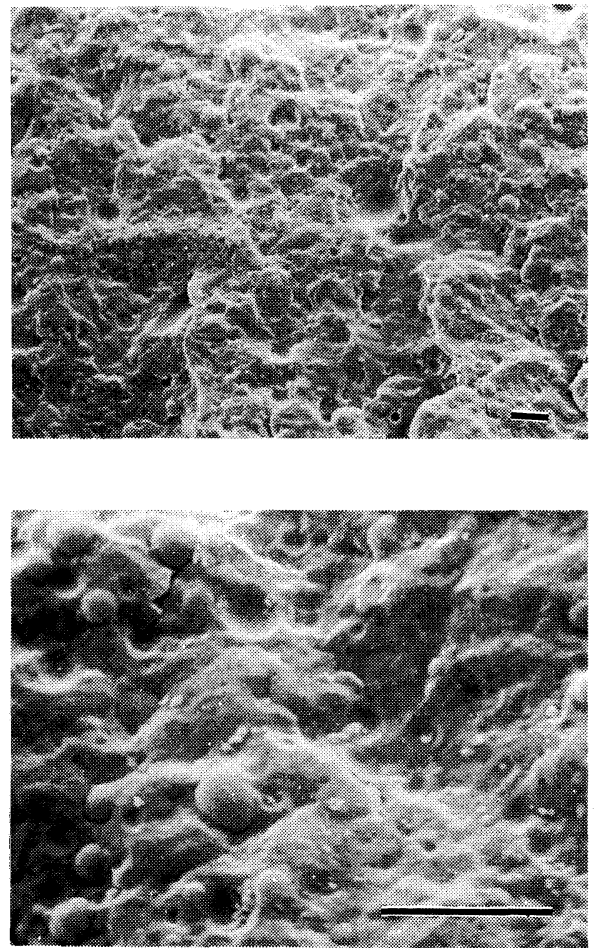

Figure 3. Scanning electron micrographs of fractured surface of the blends: A) PP/PMMA $=70 / 30 ; B$ ) PP/PMMA/block copolymer $=64 / 27 / 9$; scale bar, $10 \mu \mathrm{m}$. 
ure 1) showed two glass transitions $\left(T_{\mathrm{g}}\right)$ at near $-3^{\circ} \mathrm{C}$ and $105^{\circ} \mathrm{C}$, which might be due to $T_{\mathrm{g}}$ of polypropylene sequence and of PMMA one, respectively.

A micro-phase-separated morphology with $200-300 \AA$ dimension, which is considered to be essentially characteristic to well organized block copolymer, was observed for poly(propylene- $b$-PMMA) by staining with ruthenium tetroxide. Any domain of larger size was not observed in any part of various microtomed sections of the block copolymer. From the individual test of staining each homopolymer, the stained (black) part might be polypropylene sequence.
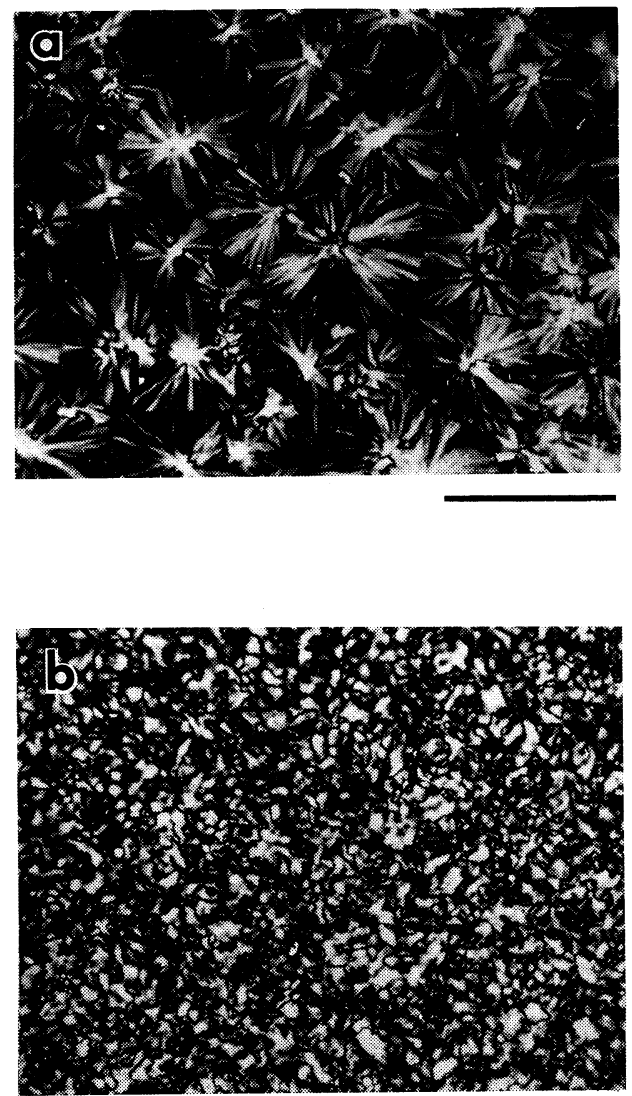

Figure 4. Polarized optical micrographs of the blends: a) PP/PMMA $=60 / 40 ;$ b) $\mathrm{PP} / \mathrm{PMMA} /$ block copolymer $=$ $55 / 36 / 9$; scale bar, $100 \mu \mathrm{m}$.

\section{Morphologies of PP/PMMA Blends}

Scanning electron micrograph of fractured surface prepared at liquid nitrogen temperature indicates a lack of interfacial adhesion in the binary blend of PP-PMMA $(70: 30)$ as shown in Figure 3A. PMMA particles seem unattached to PP matrix and leave clearly apparent holes upon cryofracture. However, small amount of the block copolymer (PPPMMA-block copolymer $=64: 27: 9)$ remarkably changes the fracture surface; PMMA particle size is enormously decreased and the particles seem to be firmly adhered to the matrix as shown in Figure 3B. The morphologies were also observed by a polarized optical micoroscope for the blend film casted from xylene solution. Figure 4 indicates that simple binary blend has a large, well organized spherulite of about $100 \mu \mathrm{m}$ in diameter, while the spherulite of ternary blend containing $9 \%$ of the block copolymer is very small $(d<10 \mu \mathrm{m})$ and not well organized. These morphological changes in the fractured surface and in PP spherulite by the addition of the block copolymer suggest that poly(propylene- $b$-MMA) effectively stabilizes the interface between immiscible polymer components, PP and PMMA. It is assumed that the added block copolymer is thermodynamically driven to PP/PMMA interface. In order to confirm the location of the block copolymer, the microtomed surface of the ternary blend was etched by $n$-heptane and

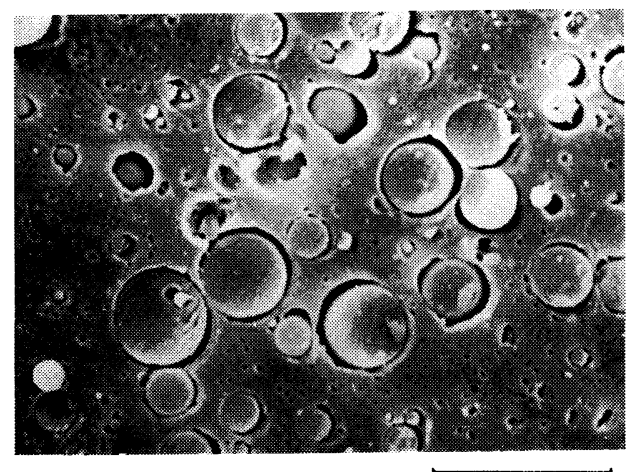

Figure 5. Solvent etched surface of the ternary blend: PP/PMMA/block copolymer $=64 / 27 / 9$; scale bar, $10 \mu \mathrm{m}$. 
observed with SEM. Only the block copolymer was extracted and neither PP nor PMMA was soluble in $n$-heptane under the condition taken here (at $60^{\circ} \mathrm{C}$ for $5 \mathrm{~min}$ ). Figure 5 clearly shows that the block copolymer forms a continuous layer around dipersed PMMA particles and its thickness is in the range of $100-1000 \AA$. Figure 5 also indicates that the part of the block copolymer disperses individually as very fine particles $(d=0.02-0.1 \mu \mathrm{m})$ in PP matrix. This result suggests that an apparent equilibrium is established for the block copolymer between the blend interface and its own domain. The phenomenon like this was also reported for the blend systems composed of PS and low-density polyethylene which was compatibilized by the triblock copolymer of PS, polyisoprene and hydrogenated polybutadine. ${ }^{28}$ Which phase the block copolymer disperses into (in this case, PP or PMMA) might depend on the chemical composition of the block copolymer. Further, how much block copolymer is necessary to stabilize an interface is another point at issue. If the copolymer of molecular weight $M$ took the ideal conformation at the interface (complete penetration of two phases), the surface occupied by the copolymer at the interface $(a)$ was derived by $\mathrm{Paul}^{29}$ as follows;

$$
a=3 \phi_{\mathrm{A}} M / R N W
$$

where $\phi_{\mathrm{A}}$ is the volume fraction of polymer $\mathrm{A}$ as apherical particles of radius $R, N$ is Avogadro's number, $W$ is the mass fraction of the block copolymer. Digital treatment of some pictures of the etched surface morphology like Figure 4 gave $R$ and $W$ to be $1.1 \mu \mathrm{m}$ and $7.5 \%$ in average, respectively (here, $W$ was taken as the fraction of the etched layer around PMMA particles). As $M=4.6 \times 10^{4}$ and $\phi_{\mathrm{A}}=0.27$, an interfacial area per copolymer molecule was calculated to be about $75 \AA^{2}$. This value is quite close to the minimum possible value of a proposed by $\mathrm{Paul}^{29}$ for the completely penetrating conformation of the diblock copolymer. Then, it can be estimated from this result that the block copolymer used in this study has sufficient cohesive forces to anchor each block sequence into each polymer component phase effectively, resulting in good adhesion at the interface.

\section{Mechanical Properties of PP/PMMA Blends}

Comparative experiments on some mechanical properties were carried out between a simple binary blend (PP-PMMA = 70:30) and a ternary blend containing $9 \%$ of the block copolymer (PP-PMMA-block copolymer= $70: 30: 10$ ). The elongation at break (EB) and the tensile impact strength (TI) were quite significantly enhanced by the added block copolymer as shown in Table III. The transformation from brittle characteristics to ductile behavior like this by the addition of

Table III. Mechanical properties of PP/PMMA blends.

\begin{tabular}{|c|c|c|c|c|c|}
\hline & \multirow{3}{*}{ Sample } & \multicolumn{3}{|c|}{ Tensile test ${ }^{\mathbf{a}}$} & \multirow{2}{*}{$\frac{\text { Tensile impact tes }}{\text { Impact strength }}$} \\
\hline & & Y.S. & U.S. & U.E. & \\
\hline & & $\mathrm{kg} \mathrm{cm}^{-2}$ & $\mathrm{~kg} \mathrm{~cm}^{-2}$ & $\%$ & $\mathrm{~kg} \mathrm{~cm} \mathrm{~cm}^{-1}$ \\
\hline 1) & PP/PMMA & 165 & 149 & 150 & 5.7 \\
\hline 2) & PP/PMMA/block & 152 & 165 & 530 & 16.9 \\
\hline 3) & PP/PMMA/h-PP & 155 & 161 & 117 & 6.4 \\
\hline
\end{tabular}

Blend compositions: sample 1) 70/30; samples 2) and 3) 64/27/9.

a Y.S, yield strength; U.S., ultimate strength; U.E., elongation at break.

b h-PP; homo-polypropylene prepared by the coordination living polymerization with the catalyst system used in this study. 
block copolymers were also reported for some blend systems. ${ }^{15,30}$ These improvements in EB and $\mathrm{TI}$ are considered to be due to the increased adhesion at the PP-PMMA interface by the block copolymer as seen in the fractured surface morphology of the ternary blend (Figure 2).

Since polypropylene sequence which is a major component $(73 \mathrm{wt} \%)$ of the block copolymer has $T_{\mathrm{g}}$ at $-3^{\circ} \mathrm{C}$ as described above and does not show any crystalline peak in DSC endothermic curve nor X-ray diffraction pattern, the block copolymer could work as an impact energy absorber (elastomer) at room temperature to give high impact strength. To confirm this point, the same mechanical tests were carried out on ternary blend of PP, PMMA, and homo-polypropylene synthesized with the above catalyst system (see Table I). It was found that the homopolypropylene component did not have any improvement effect on the elongation at break or the impact strength. Then, enhanced mechanical properties of the blend containing the block copolymer is considered to be due to the effective penetration of each block sequence of the copolymer into the blend components which results in the strong adhesion at the interface.

The syndiotactic sequence is rich in each sequence of the block copolymer as described above, while the commercial PP used in this work for polymer blends has high-isotacticity (NMR isotactic triad is more than $96 \%$ ). It is interesting that the syndiotactic-rich block copolymer effectively compatibilizes the isotactic-PP/syndiotactic-PMMA blend (syndiotacticity of PMMA used here was $86 \%$ calculated from NMR syndiotactic triad). This good compatibility might be due to the good miscibility between syndiotactic PP sequence in the block copolymer and the isotactic PP used as a blend component. The experiments to confirm this have been in progress from the viewpoints of viscoelasticity, morphologies, thermodynamics, and so on.
Acknowledgement. The authors express their appreciation to Sumitomo Chemical Co., Ltd., for permission to publish this paper.

\section{REFERENCES}

1. J. W. Barlow and D. R. Paul, Polym. Eng. Sci., 24, 525 (1984).

2. S. Wu, "Polymer Interface and Adhesion," Marcel Dekker, New York, N.Y., 1982.

3. D. W. Bartlett, J.W. Barlow, and D. R. Paul, SPE Tech. Pap., 27, 487 (1981).

4. R. Fayt, R. Jerome, and Ph. Teyssie, J. Polym. Sci., Polym. Lett. Ed., 19, 79 (1981).

5. R. Fayt, R. Jerome and Ph. Teyssie, Polym. Eng. Sci., 27, 328 (1987).

6. T. Yamamoto, Nihon Gomu Kyoukaishi, 63, 602 (1989).

7. H. Abe and S. Hosoda, Abstracts of Papers, 1st Japan SAMPE Int. Symp., 1989, p 622.

8. T. Nishio, Y. Suzuki, K. Kojima, and M. Kakugo, Koubunshi Ronbunshu, 47, 329 (1990).

9. N. Yamaguchi, T. Nambu, Y. Toyoshima, K. Mashita, and T. Ohmae, Abstracts of Papers, 5th PPS Meeting, 1989, p 86.

10. J. W. Barlow, G. Shaver, and D. R. Paul, Abstracts of Papers, Compalloy '89, 1989, p 221.

11. F. Ide and A. Hasegawa, J. Appl. Polym. Soc., 18, 963 (1974).

12. R. Fayt, R. Jerome, and Ph. Teyssie, Makromol. Chem., 187, 837 (1986).

13. L. D. Giudice, R. E. Cohen, G. Attalla, and F. Bertinotti, J. Appl. Polym. Sci., 30, 4305 (1985).

14. M. A. Drzewinski and R. E. Cohen, J. Polym. Sci., Polym. Phys. Ed., 24, 2457 (1986).

15. M. Yoshida, J. J. Ma, K. Min, J. L. White, and R. P. Quirk, Polym. Eng. Sci., 30, 30 (1990).

16. R. Fayt, R. Jerome, and Ph. Teyssie, Polym. Eng. Sci., 27, 328 (1987).

17. R. Fayt and Ph. Teyssie, Macromolecules, 19, 2077 (1986).

18. T. Ouhadai, R. Fayt, R. Jerome, and Ph. Teyssie, Polym. Commun., 27, 212 (1986).

19. Ph. Teyssie, R. Fayt, and R. Jerome, Polym. Mater. Sci. Eng., 58, 622 (1988).

20. J. Heuschen, J. M. Vion, R. Jerome, and Ph. Teyssie, Polymer, 31, 1473 (1990).

21. R. Fayt, R. Jerome, and Ph. Teyssie, J. Polym. Sci., Polym. Chem. Ed., 27, 2823 (1989).

22. T. Ouhadi, R. Fayt, R. Jerome, and Ph. Teyssie, $J$. Appl. Polym. Sci., 32, 5647 (1986).

23. T. Ouhadi, R. Fayt, R. Jerome, and Ph. Teyssie, $J$. Polym. Sci., Polym. Phys. Ed., 24, 973 (1986).

24. Y. Doi, S. Ueki, and T. Keii, Makromol. Chem., Rapid Commun., 2, 225 (1982).

25. Y. Doi, Y. Watanabe, and K. Soga, Makromol. 
Chem., Rapid Commun., 4, 533 (1983).

26. Y. Doi, T. Koyama, and K. Soga, Makromol. Chem., 186, 11 (1985).

27. Y. Doi, S. Suzuki, and K. Soga, Makromol. Chem., Rapid Commun., 6, 639 (1985).

28. R. Fayt, R. Jerome, and Ph. Teyssie, J. Polym. Sci.,
Polym. Lett. Ed., 24, 25 (1986).

29. D. R. Paul and S. Newman, "Polymer Blends," Vol.

2, Academic Press, New York, N.Y., 1978.

30. C. R. Lindsey, D. R. Paul, and J. W. Barlow, J. Appl. Polym. Sci., 26, 1 (1981). 